\title{
The recognition and management of sepsis and septic shock: a guide for non-intensivists
}

\author{
Alexander Keeley, Paul Hine, Emmanuel Nsutebu
}

Tropical and Infectious Disease Unit, Royal Liverpool University Hospital, Liverpool, UK

\section{Correspondence to}

Dr Emmanuel Nsutebu, Tropical and Infectious Disease Unit, Royal Liverpool University Hospital, Liverpool L7 8XP, UK; nsutebufru@hotmail.com

Received 10 September 2016 Revised 28 April 2017

Accepted 30 April 2017 Published Online First 29 July 2017
CrossMark

To cite: Keeley A, Hine $P$, Nsutebu E. Postgrad Med J 2017;93:626-634.

\section{ABSTRACT}

Sepsis is common, often fatal and requires rapid interventions to improve outcomes. While the optimal management of sepsis in the intensive care setting is the focus of extensive research interest, the mainstay of the recognition and initial management of sepsis will occur outside the intensive care setting. Therefore, it is key that institutions and clinicians remain well informed of the current updates in sepsis management and continue to use them to deliver appropriate and timely interventions to enhance patient survival. This review discusses the latest updates in sepsis care including the new consensus definition of sepsis, the outcome of the proCESS, ProMISe and ARISE trials of early goal directed therapy (EGDT), and the most recent guidelines from the Surviving Sepsis Campaign.

\section{INTRODUCTION}

Sepsis is common and often fatal, representing a major public health problem. Estimates of the incidence of sepsis vary widely due to differences in case ascertainment, ranging from 66 to 300 per 100000 population in the developed world. ${ }^{12}$ However, there is consensus that the incidence is increasing, driven by an ageing population with multiple comorbidities, increased use of immunosuppressive therapy and high-risk interventions. ${ }^{3} 4$ Mortality estimates for sepsis range from $27 \%$ to $36 \%{ }^{4-6}$; however, the risk of death from sepsis has been falling over recent decades despite the increasing incidence, perhaps due to improvements in care. ${ }^{7}$

The Third International Consensus Definitions for Sepsis and Septic Shock (Sepsis-3) were published in February 2016. The new definitions are intended to improve the clarity of the definitions for clinical care, epidemiology, quality improvement and research. The new definitions have been validated retrospectively by using large databases in the USA and Germany. ${ }^{8}$

The pathophysiology of sepsis is incompletely understood. There appears to be inappropriate regulation of normal physiological reactions to infection, both pro-inflammatory and anti-inflammatory, resulting in life-threatening organ dysfunction. ${ }^{9} 10$

The Surviving Sepsis Campaign (SSC) has been a key influence on sepsis care and is a unique international collaboration for quality improvement which was established in 2002 by the European Society of Critical Care Medicine, the International Sepsis Forum and the Society of Critical Care Medicine. The SSC has developed evidence-based guidelines for the management of sepsis, in tandem with interventions to change bedside practice. ${ }^{11-15}$ The key recommendations are shown in box 1 :

The recent guidelines updated in 2016 are shown in box 1 .

The UK Sepsis Trust has developed an initiative called the 'Sepsis Six' designed to facilitate the delivery of the SSC resuscitation bundle. The Sepsis Six bundle (box 2) is designed to be completed within 1 hour and includes simple measures for assessment, resuscitation and risk stratification, which can be implemented at the bedside by nurses and doctors.

Early goal directed therapy (EGDT) is a quantitative resuscitation protocol which sets physiological targets for resuscitation in order to restore tissue perfusion in patients with septic shock. It was first successfully trialled by Rivers and collaborators in $2001,{ }^{16}$ and formed the basis of the 6-hour bundle of the SSC. More recently, three large multicentre randomised controlled studies, the Protocolised Care for Early Septic Shock (ProCESS), ${ }^{17}$ The Australasian Resuscitation in Sepsis Evaluation (ARISE) trials ${ }^{18}$ and the Protocolised Management in Sepsis (ProMISe) trial did not demonstrate outcome benefit from EGDT compared with usual care in patients with septic shock. ${ }^{19}$ The new SSC guidelines de-emphasise the protocolisation of care and invasive monitoring. They recommend that patients should be re-evaluated frequently by clinicians. ${ }^{15}$ Data from a prospective cohort study from the SSC showed that compliance with SSC bundles led to a $25 \%$ relative risk reduction in mortality. ${ }^{14} \mathrm{~A}$ review of bundled care for septic shock has shown consistent improvement in survival. ${ }^{20}$ Therefore, although the effectiveness of EGDT is now uncertain, there is little doubt that sepsis bundles improve outcomes. The key clinical question is which components of sepsis care are most important. The aim of this review is to update the reader on the latest definition of sepsis and the implications of the ProCESS, ARISE and ProMISe trials. It is also intended to assist non-intensivist medical professionals in recognising cases of sepsis and ensuring that the management of patients is appropriate and optimal.

\section{DEFINITIONS}

\section{A new definition for sepsis}

In February 2016, the Third International Consensus Definitions for Sepsis and Septic Shock (Sepsis-3) were published. The Sepsis-3 task force redefined the definitions of sepsis (removing severe sepsis from the definitions) and septic shock. The purpose of this work was to improve the clarity of the definitions not only for the clinical care of 
Box 1 The Surviving Sepsis Campaign 2016 key

recommendations

- Intravenous antibiotics should be started within 1 hour of sepsis recognition and should include combination therapy (at least two classes of antibiotics to cover a known or suspected pathogen) for patients with septic shock. Combination therapy should not routinely be used for patients without shock.

- Patients with hypoperfusion should receive at least $30 \mathrm{~mL} /$ $\mathrm{kg}$ of intravenous crystalloid within 3 hours and should be re-assessed frequently.

- For patients who require vasopressors, the initial target mean arterial pressure (MAP) should be $65 \mathrm{~mm} \mathrm{Hg}$.

- Norepinephrine is the first choice for patients who need vasopressors. Vasopressin or epinephrine can be added. For patients who remain unstable, dobutamine is recommended.

- Intravenous hydrocortisone ( $200 \mathrm{mg} /$ day) is suggested for patients who are haemodynamically unstable despite fluids and vasopressors.

- Blood transfusion should be reserved for patients with a haemoglobin concentration of $<7.0 \mathrm{~g} / \mathrm{dL}$, except in special circumstances such as haemorrhage and myocardial ischaemia. Platelets should be given if the platelet count is $<10000 / \mathrm{mm}^{3}$ or $<20000 / \mathrm{mm}^{3}$ with bleeding.

- Sodium bicarbonate should not be used for most patients with $\mathrm{pH} \geq 7.15$.

patients but also for epidemiology, quality improvement and research. The new definitions are also intended to ensure that the word sepsis is used in a consistent manner. The criteria have been validated retrospectively by using large databases in the USA and Germany. ${ }^{8}$

Sepsis is now defined as 'life threatening organ dysfunction caused by dysregulated host response to infection'. The clinical diagnosis of sepsis is based on a patient having infection and a raised Sequential (Sepsis-related) Organ Failure Assessment Score (SOFA), with a change in score of 2 or greater from baseline being diagnostic (table 1).

The definition can be used to identify patients who have an in-hospital mortality of $>10 \%$ and a 2 to 25 -fold increased risk of mortality compared with patients with a SOFA score of $<2$. In summary, the new definitions are designed to identify the sickest patients and those who benefit from prompt intervention and in whom empirical broad spectrum antibiotic therapy is warranted. The term 'severe sepsis' should no longer be employed. However, SOFA scoring is not yet used widely in critical care units and is currently rarely used in emergency departments and wards.

Box 2 Sepsis Six management bundle to be implemented within 1 hour of onset of sepsis

Sepsis Six bundle to be completed within 1 hour:

- Administer oxygen to maintain $\mathrm{SpO}_{2}$ at $>94 \%$.

- Take blood cultures and consider infective source.

- Administer intravenous antibiotics.

- Consider intravenous fluid resuscitation.

- Check serial lactates.

- Commence hourly urine output measurement.
Septic shock is defined as a 'subset of sepsis with particularly profound circulatory, cellular and metabolic abnormalities associated with a greater risk of mortality than sepsis alone'. The diagnostic criteria of septic shock are a 'Vasopressor requirement required to maintain a MAP of $>65 \mathrm{~mm} \mathrm{Hg}$ and a serum lactate level $>2 \mathrm{mmol} / \mathrm{L}$ in the absence of hypovolaemia'. ${ }^{8}$ The new definition for septic shock identifies patients with an in-hospital mortality of $>40 \%$.

\section{The qSOFA score and sepsis}

The SOFA score is complex and the information required to calculate the score may not be available when the patient is acutely unwell. As a result, the qSOFA (quick SOFA) score was developed as a bedside tool to rapidly identify adult patients with infection who are more likely to have poor outcomes. qSOFA is considered to be positive if the patient has at least two of the following clinical criteria:

- Respiratory rate of $22 / \mathrm{min}$ or greater,

- Altered mentation (Glasgow Coma Scale of $<15$ ), or

- Systolic blood pressure of $100 \mathrm{~mm} \mathrm{Hg}$ or less.

The task force recommend use of qSOFA to prompt clinicians to:

- Further investigate for organ dysfunction,

- Initiate or escalate therapy as appropriate, and

- Consider referral to critical care or increase the frequency of monitoring, if such actions have not already been undertaken.

The task force considered that positive qSOFA criteria should also prompt consideration of possible infection in patients not previously recognised as infected (similar to how SIRS criteria were used previously).

Publication of the Sepsis-3 definitions resulted in concerns that they may delay identification and treatment of sepsis. Prospective assessment of these definitions is required. In the mean time, hospitals and sepsis programmes such the SSC have produced recommendations and statements which clinicians should refer to. ${ }^{21}$

\section{Systemic inflammatory response syndrome and sepsis}

Systemic inflammatory response syndrome (SIRS) is a pro-inflammatory state, usually but not necessarily, caused by infection. SIRS criteria (box 3) are no longer required for the diagnosis of sepsis. As approximately 10\%-12\% of patients with sepsis in intensive care units may not have $\geq 2$ SIRS criteria, using the SIRS criteria alone will not identify all patients with sepsis. ${ }^{22}$ However, the presence of a systemic inflammatory response may still have an important role in identifying patients with infection.

\section{Early warning scores and sepsis}

Many clinicians now use track-and-trigger early warning score systems as a standard of care to identify patients at risk of deteriorating. Two examples of these scores are the National Early Warning Score (NEWS) and the Modified Early Warning Score (MEWS) used in the UK (table 2). The scores are validated tools for predicting poor outcomes and are commonly used in healthcare facilities.

A retrospective single centre study in 2015 showed that a NEWS score of 3 or more in the emergency department has a sensitivity of $92.6 \%$ (95\% CI $74.2 \%$ to $98.7 \%$ ) and a specificity of $77 \%(95 \%$ CI $72.8 \%$ to $80.6 \%)$ to detect patients at risk of sepsis and septic shock at triage. ${ }^{23}$ Another retrospective 
Table 1 Sequential (Sepsis-related) Organ Failure Assessment Score. ${ }^{8}$

\begin{tabular}{|c|c|c|c|c|c|}
\hline & Score & & & & \\
\hline System & 0 & 1 & 2 & 3 & 4 \\
\hline \multicolumn{6}{|l|}{ Respiration } \\
\hline $\mathrm{PaO}_{2} / \mathrm{FiO}_{2}, \mathrm{kPa}$ & $\geq 53.3$ & $<53.3$ & $<40$ & $<26.7$ with respiratory support & $<13.3$ with respiratory support \\
\hline \multicolumn{6}{|l|}{ Coagulation } \\
\hline Platelets, $\times 10^{9} / \mathrm{L}$ & $\geq 150$ & $<150$ & $<100$ & $<50$ & $<20$ \\
\hline \multicolumn{6}{|l|}{ Liver } \\
\hline Bilirubin, $\mu \mathrm{mol} / \mathrm{L}$ & $<20$ & $20-32$ & 33-101 & $102-204$ & $>204$ \\
\hline \multicolumn{6}{|l|}{ Cardiovascular } \\
\hline $\mathrm{MAP}, \mathrm{mm} \mathrm{Hg}$ & $\geq 70$ & $<70$ & & & \\
\hline $\begin{array}{l}\text { Catacholamine dose, } \mu \mathrm{g} / \\
\mathrm{kg} / \mathrm{min}\end{array}$ & & & Dopamine $<5$ or dobutamine & $\begin{array}{l}\text { Dopamine } 5.1-15 \text { or epinephrine } \leq 0.1 \\
\text { or norepinephrine } \leq 0.1\end{array}$ & $\begin{array}{l}\text { Dopamine }>15 \text { or epinephrine }>0.1 \text { or } \\
\text { norepinephrine }>0.1\end{array}$ \\
\hline \multicolumn{6}{|l|}{ Central nervous system } \\
\hline GCS & 15 & $13-14$ & $10-12$ & $6-9$ & $<6$ \\
\hline \multicolumn{6}{|l|}{ Renal } \\
\hline Creatinine, $\mu \mathrm{mol} / \mathrm{L}$ & $<110$ & $110-170$ & $171-299$ & $300-440$ & $>440$ \\
\hline Urine output, ml/day & & & & $<500$ & $<200$ \\
\hline
\end{tabular}

GCS, Glasgow Coma Scale; MAP, mean arterial pressure.

A change in score of 2 or more in the presence of infection is a diagnosis of sepsis. This must prompt the clinician to initiate sepsis management bundles. Reproduced from Singer et al. ${ }^{8}$

study published in 2013 showed that a single early warning score of 5 or above in the emergency department can be useful in predicting mortality in patients with sepsis. ${ }^{24}$ In addition, a recent large retrospective study showed that MEWS and NEWS scores were more sensitive for predicting death or ICU transfer. ${ }^{25}$ For example, at the same level of specificity, a NEWS score of 7 or above was $13 \%$ more sensitive than qSOFA. It is not surprising that early warning scores appear to outperform qSOFA as they contain most elements of the qSOFA score and other additional parameters to assess deterioration. It is likely that in the coming years, frontline clinicians may use aggregate early warning scores in identifying and treating patients with sepsis.

A reliable operational sepsis definition for use by frontline clinicians is urgently required. While the various options such as SIRS, qSOFA and early warning scores are being evaluated, front line clinicians should use which ever tool is recommended by their institution or local sepsis teams.

\section{RECOGNITION OF SEPSIS}

The window between the onset and identification of sepsis is often where significant delays in management occur and where appropriate management is crucial. ${ }^{26}$ Rapid recognition and resuscitation of patients with sepsis is therefore key to the effective management of sepsis. Deteriorating patients with a raised early warning score (such as a raised aggregate early warning score of 5 or above) should therefore be screened for infection.

\section{Box 3 Criteria for a patient to meet SIRS}

- Temperature $>38^{\circ} \mathrm{C}$ or $<36^{\circ} \mathrm{C}$

- Respiratory rate $>20$ breaths per minute

- Heart rate $>90$ beats per minute

- White blood cell count of $>12 \times 10^{9} / \mathrm{L}$ or $<4 \times 10^{9} / \mathrm{L}$

Note: In order to meet SIRS criteria a patient must have two or more of these variable. SIRS criteria should prompt the clinician to assess for sepsis and septic shock and apply initial management.

\section{INVESTIGATIONS}

When faced with a patient with sepsis, initial investigations to identify end organ hypoperfusion and identify the source of infection are important. Antibiotics should only be administered immediately when there is likely to be delay in obtaining samples for culture; however, it is nearly always possible to obtain blood cultures before administering antibiotics.

\section{Investigations to assess for end organ hypoperfusion}

Investigations include:

- Full blood count

- Clotting profile, with prothrombin time and INR

- Urea and electrolytes

- Liver function tests

- Serum lactate

- Arterial blood gas measurement.

These investigations should be performed and results reviewed without delay. Most clinical areas have access to laboratory lactate measurement or arterial blood gas machines capable of providing a rapid arterial or venous lactate result.

\section{Lactate}

An increasing body of evidence indicates that lactate is a useful predictor of outcome in sepsis. Results from the Surviving Sepsis Campaign database showed that patients with lactate values $>4 \mathrm{mmol} / \mathrm{L}$ had significantly increased mortality, and that there is a linear relationship between lactate and mortality, confirming earlier findings. ${ }^{27}{ }^{28}$ Lactate may be used to identify patients with 'cryptic septic shock', those who have end organ hypoperfusion without hypotension due to compensation. Recognition of hypoperfusion in these patients might otherwise be delayed, leading to poorer outcomes. ${ }^{29}$ A baseline lactate also allows the targeting of resuscitation towards lactate clearance, which is a good prognostic marker. For these reasons, serum lactate represents an easy, rapid and useful tool for risk stratification for the non-intensivist at first point of contact with the patient with sepsis. It has become integral to SSC guidance to ensure recognition of hypoperfusion and appropriate management. 
Table 2 Modified Early Warning Score (MEWS) and National Early Warning Score (NEWS) used to identify patients at risk of deterioration

\begin{tabular}{|c|c|c|c|c|c|c|c|}
\hline Physiological parameter & Score & & & & & & \\
\hline & 3 & 2 & 1 & 0 & 1 & 2 & 3 \\
\hline \multicolumn{8}{|l|}{ Modified Early Warning Score } \\
\hline Respiratory rate & & $\leq 8$ & & $9-12$ & $15-20$ & $21-29$ & $>29$ \\
\hline Heart rate & & $\leq 40$ & $41-50$ & $51-100$ & $101-110$ & $111-129$ & $>129$ \\
\hline Systolic BP (mm Hg) & $\leq 70$ & $71-80$ & $81-100$ & 101-199 & & $\geq 200$ & \\
\hline Urine output (mL/kg/hour) & Nil & $<0.5$ & & & & & \\
\hline Temperature & & $\leq 35$ & $35.1-36$ & $36.1-38$ & $38.1-38.5$ & $\geq 38.6$ & \\
\hline Level of consciousness (AVPU scale) & & & & A & V & $P$ & $u$ \\
\hline \multicolumn{8}{|l|}{ National Early Warning Score } \\
\hline Respiratory rate & $\leq 8$ & & $9-11$ & $12-20$ & & $21-24$ & $\geq 25$ \\
\hline Oxygen saturations (\%) & $\leq 91$ & $92-93$ & $94-95$ & $\geq 96$ & & & \\
\hline Supplemental oxygen & & Yes & & No & & & \\
\hline Temperature & $\leq 35$ & & $35.1-36$ & $36.1-38.0$ & $38.1-39$ & $\geq 39.1$ & \\
\hline Systolic BP (mm Hg) & $\leq 90$ & $91-100$ & $101-110$ & $111-219$ & & & $\geq 220$ \\
\hline Heart rate & $\leq 40$ & & $41-50$ & $51-90$ & $91-110$ & $111-130$ & $\geq 131$ \\
\hline Level of consciousness (AVPU scale) & & & & A & & & $\mathrm{V}, \mathrm{P}, \mathrm{U}$ \\
\hline
\end{tabular}

AVPU scale: $A$, alert; $V$, response to voice only; $P$, response to pain only; $U$, unconscious.

\section{Obtaining microbiological samples}

SSC guidelines recognise the importance of obtaining appropriate cultures before antimicrobial therapy, to allow confirmation of infection, identification of pathogens, and subsequent de-escalation of antimicrobial therapy. SSC guidelines recommend taking two sets of blood cultures. A second set of blood culture increases the sensitivity for the detection of bacteraemia from approximately $70 \%$ to $90 \%$, although estimates vary. ${ }^{30} 31$ Culture and determination of the susceptibility profile provides information required to de-escalate or rationalise antimicrobial therapy, a key component of antimicrobial stewardship. ${ }^{32}$ Obtaining blood cultures after antibiotic administration significantly reduces their sensitivity and clinical utility. However, obtaining cultures before antimicrobial therapy can be challenging, particularly in view of the recommendation from Sepsis Six to administer antibiotics within 1 hour of sepsis recognition. ${ }^{33}$ There are currently blood culture media which use resins and charcoal to inactivate antibiotics in the case of unacceptable delays in the administration of antibiotics before samples are taken. ${ }^{34}$ In addition to blood cultures, other samples should be collected for culture when clinically indicated, but this should not delay antibiotic administration. ${ }^{35}$

\section{INITIAL CLINICAL MANAGEMENT OF SEPSIS}

The therapeutic goals in the management of sepsis are to improve tissue oxygenation and perfusion, and to provide antimicrobial therapy with suitable cover against the causative organism.

\section{Antibiotics}

Timing of antibiotics

The SSC has advocated early antibiotic therapy since its inception, and the recommendation has been supported by compelling evidence. ${ }^{36}$ The updated 2016 SSC guidelines recommend intravenous antibiotics are administered within 1 hour of recognition of sepsis. ${ }^{15}$ In 2014 a large retrospective study of SSC data confirmed that delay in antibiotic administration is associated with increased in-hospital mortality, and for each hour delay there is a linear increase in risk of mortality. ${ }^{37}$ This was the largest study published so far and assessed time from the development of sepsis to the administration of antibiotics in patients in critical care, general wards and emergency departments. Early antibiotic administration is therefore arguably the single component of the SSC for which the most compelling evidence exists. $^{20}$

\section{Practical considerations}

There are a number of practical concerns within the SSC guidelines that are of particular relevance to the non-intensivist:

- Additional vascular access ports may be required to facilitate prompt infusion alongside fluid resuscitation.

- Clinicians should be aware that some antimicrobial agents can be given as a bolus infusion, for example, meropenem and teicoplanin. If vascular access is limited and other agents are being infused, this may offer an advantage.

\section{Choice of antimicrobials}

Antimicrobial agents should be chosen to treat likely causative pathogens. Clinicians should follow local guidelines, which should also be based on local antimicrobial resistance patterns.

The empiric choice of antimicrobials may need to cover resistant organisms such as:

- Methicillin-resistant Staphylococcus aureus, for which a single genetic mutation confers resistance to the commonly prescribed $\beta$-lactam antimicrobials.

- Vancomycin-resistant enterococci. These occur in outbreaks; infections are difficult to treat and colonisation is difficult to eradicate. $^{38}$

- Gram-negative bacteria, in particular Klebsiella pneumonia and Escherichia coli, which produce extended spectrum $\beta$-lactamases (ESBL), enzymes that deactivate $\beta$-lactam antibiotics. ${ }^{39}$ Emergence of ESBL has led to increasing use of carbapenem $\beta$-lactam antibiotics. In turn this has led to the emergence of multi-resistant bacteria which produce carbapenemase enzymes, collectively known as carbapenemase-producing enterobacteriaceae (or CPE).

A major challenge for clinicians treating patients with sepsis and septic shock is to ensure that the antimicrobial choice provides a broad enough spectrum of cover to treat all likely causative organisms. Endogenous infections caused by colonising bacteria are common and should also be considered. It is therefore 
important to be aware of risk factors for antimicrobial resistance, such as previous antimicrobial therapy, previous hospitalisation and nursing home residency. A thorough antimicrobial treatment history and review of clinical records is often valuable. Review of previous microbiology results is very important and recently used antimicrobials should be avoided. Combination antimicrobial therapy to provide broad spectrum cover may be required and is encouraged in patients colonised with multi-resistant pathogens and in immunosuppressed patients. ${ }^{5}$ Combination antimicrobial therapy is also recommended in patients with septic shock (SSC 2016 guideline reference).

\section{Intravenous fluids}

Type of fluid

Initial fluid resuscitation should be with crystalloids. There is no convincing evidence to suggest the superiority of any alternative. Colloid solutions may be associated with increased risk of acute kidney injury and a marginal benefit has been observed for resuscitation with albumin-containing solutions. ${ }^{40-42}$ Crystalloids are cheaper and more readily available. Thus SSC recommends using crystalloid initially and that albumin-containing solutions can be used in patients who have received large volumes of crystalloid. ${ }^{5}$

\section{Volume of fluid}

In patients with hypoperfusion, a minimum of $30 \mathrm{~mL} / \mathrm{kg}$ of intravenous crystalloid fluid should be administered as a fluid challenge within 3 hours. For an average $70 \mathrm{~kg}$ patient, this means a $2.1 \mathrm{~L}$ fluid challenge initially. There is no evidence to suggest the optimum volume of fluid to administer. The response to initial resuscitation should determine the total volume administered. Patients enrolled in the EGDT trials including ProCESS, ARISE and ProMISe, received on average $2-5 \mathrm{~L}$ of fluid within the first 6 hours. ${ }^{16-19}$ The SSC recommends a conservative fluid strategy for patients with sepsis-induced acute lung injury (pulmonary oedema). A recent small retrospective study has shown that fluid overload in sepsis may be associated with poorer outcomes. ${ }^{43}$ It is therefore important to monitor response to fluids to prevent fluid overload or worsening of sepsis-induced acute lung injury.

\section{Source control}

Source control involves measures undertaken to eliminate a focus of infection, to control ongoing contamination, and to restore premorbid anatomy and function. ${ }^{44}$ SSC guidelines recommend that this should take place within the first 12 hours after diagnosis, and the least invasive procedure should be used. This may include drainage of infected fluid collections, debridement of infected solid tissue, and removal of devices and foreign bodies including intravascular access devices or surgery. ${ }^{45}$

The procedure used for source control will vary depending on the site and type of infection, risks and local resources. ${ }^{46}$

Available evidence for the timing of source control comes largely from retrospective cohort studies, and suggests better outcomes with earlier intervention. An example is a recent large Danish study showing that in perforated peptic ulcer cases, each hour of delay from admission to surgery was associated with increased risk of mortality. ${ }^{47}$ A much smaller prospective study has demonstrated this specifically in the context of septic shock. ${ }^{48}$ A key exception, highlighted in SSC guidelines, is the case of peripancreatic necrosis, in which a delayed approach is preferred.

\section{Monitoring response to treatment}

Traditionally, EGDT has been used to mean intensive-care-based protocolised management to meet the following physiological targets:

- Mean arterial pressure of $65 \mathrm{~mm} \mathrm{Hg}$

- Urine output of $0.5 \mathrm{~mL} / \mathrm{kg} / \mathrm{hour}$

- Central venous pressure of 8-12 mm Hg

- Superior vena cava oxygen saturation of $70 \%$ or mixed venous oxygen saturation of $65 \%$.

The initial rationale for EGDT was derived from a single-centre trial showing dramatic improvement in mortality rates $(30.5 \%$ vs $46.5 \%$ ) in patients with septic shock managed with protocolised care to meet the above physiological targets. ${ }^{16}$

However, recently published results from three large multicentre trials show no difference in outcomes between EGDT and usual care. The ProCESS trial in the USA of patients with septic shock showed no difference in 60-day mortality between groups managed to targets derived from invasive monitoring with EGDT, versus those managed to a protocol which did not require central monitoring, or by usual care. ${ }^{17}$ This study was carried out in academic centres and the overall mortality of $19 \%$ is far lower than classically observed for septic shock. All groups received on average more than $2 \mathrm{~L}$ of initial fluid resuscitation and $75 \%$ of patients had received antibiotics prior to randomisation. Similarly, the ARISE multicentre randomised trail compared EGDT to usual care in Australia and New Zealand and showed no difference in 90-day mortality, with an overall mortality rate of $18.7 \%{ }^{18}$ Results of the ProMISe multicentre randomised trial have shown no difference in outcomes of EGDT versus usual care in UK NHS hospitals, with overall mortality of $29.4 \% .{ }^{19}$ Collaborative meta-analysis of all three trials also showed no difference between EGDT and usual care. ${ }^{49}$ The 2016 updated SSC guideline emphasises the benefits of early identification and resuscitation in sepsis and septic shock. The guidelines also stress the importance of frequent re-evaluation by clinicians and de-emphasises protocolisation and invasive monitoring. ${ }^{15}$

It is important to stress the importance of close monitoring of initial response to treatment, using non-invasive assessments of tissue hypoperfusion. For the non-intensivist, the hourly monitoring of urine output, combined with blood pressure response to fluid therapy, is an essential clinical tool to guide ongoing resuscitation. Failure to meet SSC targets with intravenous fluids should prompt referral to critical care services.

\section{Lactate monitoring}

Trials have shown that patients who clear raised lactate levels have improved outcomes, and the slower the clearance, the poorer the outcome. ${ }^{5051}$ Therefore, resuscitation can be targeted to the normalisation of serum lactate, obtained by serial venous measurement. ${ }^{52}$ In a non-intensive care setting, re-measurement of lactate can identify those not responding to intravenous fluid resuscitation. While lactate may be non-inferior to monitoring of central venous oxygen saturations in intensive care settings, a recent open-label study comparing serial lactate monitoring to standard intensive care management (with no knowledge of serial lactate values) showed improvement in survival, as well as a shortened intensive care stay for the lactate group. ${ }^{50} 53$

\section{Airway support and oxygen}

One of the priorities in managing a patient with a critical illness is to stabilise the airway, particularly in a patient with a depressed level of consciousness, and to ensure adequate tissue 
oxygenation. The use of empirical high flow oxygen is no longer recommended in the Sepsis Six bundle, and oxygen should be used to keep oxygen saturations at $>94 \%$, unless there is a history or clinical suspicion of chronic obstructive pulmonary disease and type II respiratory failure, in which case a lower oxygen target should be used.

\section{FURTHER CLINICAL MANAGEMENT OF SEPSIS}

Patients in whom physiological targets cannot be met with intravenous fluid alone, may require additional management to optimise end organ perfusion. In reality this will usually be provided in a critical care setting.

\section{Vasopressors and inotropes}

Vasopressors increase blood pressure by increasing peripheral vascular resistance. In patients who remain hypotensive despite adequate fluid resuscitation, or in those who develop cardiogenic pulmonary oedema, a vasopressor should be used. Inotropes increase cardiac output by increasing cardiac contractility. They may be initiated in cases of myocardial dysfunction, or in cases of failure to achieve end organ perfusion despite adequate intravascular fluid status and MAP. ${ }^{5}$ Norepinephrine is the first choice for patients who need vasopressors. Vasopressin or epinephrine can be added. For patients who remain unstable, dobutamine is recommended. ${ }^{15}$ Use of vasopressors and introptes should be limited to critical care.

\section{Corticosteroids}

Hydrocortisone as an adjuvant therapy may have a role in patients with septic shock who fail to achieve adequate MAP despite adequate intravenous fluid and vasopressors. ${ }^{5} 54$ However, its indiscriminate use in all patients with septic shock showed no benefit in the large multicentre CORTICUS study. ${ }^{56}$ There is conflicting evidence from meta-analysis of RCTs regarding the role of low-dose corticosteroids in the treatment of all patients with septic shock, and further high quality RCTs are required to clarify their role.

\section{Transfusion of blood products}

Patients with sepsis should receive red cell transfusion when haemoglobin falls below $<7 \mathrm{~g} / \mathrm{dL}$. This follows evidence from a large multicentre trial comparing a restrictive $(<7 \mathrm{~g} / \mathrm{dL})$ with a liberal $(<9 \mathrm{~g} / \mathrm{dL})$ transfusion threshold. ${ }^{57}$ Patients with active bleeding, myocardial ischaemia or severe hypoxia are exceptions and should be transfused at higher haemoglobin thresholds. ${ }^{5}$ Fresh frozen plasma should not be used to correct documentable coagulopathy unless there is intercurrent bleeding or invasive procedures are planned. ${ }^{5}$ The threshold for transfusion of platelets should be $<10 \times 10^{9} / \mathrm{L}$ in cases where there is no bleeding risk, $<20 \times 10^{9} / \mathrm{L}$ if there is bleeding risk or the patient is receiving chemotherapy and $<50 \times 10^{9} / \mathrm{L}$ if invasive procedures are planned.

\section{Glucose control}

There is lack of clear-cut evidence regarding targets for optimal control of glucose with insulin in patients with sepsis, partly because of the large variety of patient factors and variation in glucose protocols from various RCTs. ${ }^{58}$ It is becoming increasingly clear from a series of meta-analyses that aiming for intensive control of blood glucose $(<6.1 \mathrm{mmol} / \mathrm{L})$ confers no survival benefit. ${ }^{59-62}$ In fact, the most robust study (NICESUGAR) comparing intensive blood glucose control with standard control with insulin, using a cut-off blood glucose for insulin therapy of $10 \mathrm{mmol} / \mathrm{L}$, showed increased mortality in the intensive insulin group. ${ }^{63}$ SSC therefore recommends intervention (with an insulin protocol) to maintain glucose at a cut-off value of $10 \mathrm{mmol} / \mathrm{L}$ in sepsis and to avoid hypoglycaemia and rapid glucose fluctuations. Serum glucose should be monitored every 1 to 2 hours until it is stable, and every 4 hours thereafter. ${ }^{5}$

\section{Additional care}

As with any critically ill patient, attention to basic patient care is important. In sepsis particular care should be taken to ensure a patient receives venous thromboembolism (VTE) prophylaxis, stress ulcer prophylaxis with a proton pump inhibitor in those with bleeding risk (ie, coagulopathy, prolonged hypotension, mechanical ventilation) and prevention of pressure ulcers. ${ }^{64-67}$

\section{THE ROLE OF THE NON-INTENSIVIST IN SEPSIS MANAGEMENT}

The non-intensivist plays a crucial role in ensuring that patients with sepsis are managed in a timely and appropriate manner. This starts with timely recognition of sepsis by recognising patients who are at risk of sepsis and screening for sepsis in patients who are unwell or suspected of having an infection. The non-intensivist should also initiate investigations to assess for end organ hypoperfusion and confirm the diagnosis. In addition, resuscitation with fluids and intravenous antibiotics should be administered within 1 hour, and liaison with critical care teams is required. All this is often expected to take place in the context of busy clinical units with multiple other pressures on a doctor's time. However, there is no doubt that rapid intervention is likely to benefit the patient. ${ }^{26}$

In order to speed up timely recognition and management of a patient with sepsis, the UK Sepsis Trust have created the Sepsis Six bundle to be implemented within 1 hour of recognition of sepsis. The six tasks are simple, practical management steps that can be implemented by a doctor or nurse in any hospital environment. The six components (listed in box 2) cover the fundamental initial steps in resuscitation and risk stratification of a patient with sepsis. No published trials have assessed the efficacy of the Sepsis Six bundle in terms of improving mortality. However, observational studies suggest it plays a useful role in the protocolisation of life-saving interventions and can improve mortality. $^{26}$

Hospitals should ensure that all medical staff are familiar with the diagnostic criteria for sepsis, and know when and how to use the SSC and Sepsis Six bundles. Hospitals should also facilitate the implementation of these bundles through the use of screening tools and pathways. ${ }^{68}$

\section{BARRIERS TO EFFECTIVE SEPSIS CARE}

Despite clear guidance dictating best practice in the management of sepsis, there are considerable barriers to implementing SSC guidance. ${ }^{26} 6869$ The crucial first step is recognition of sepsis. The process of matching abnormal parameters with a diagnosis of infection and subsequently sepsis can be difficult, and can lead to delays in commencing optimal management. ${ }^{68}$ Use of the SOFA score to diagnose sepsis and septic shock requires laboratory investigations and critical care treatment, and this may introduce further delays in a busy hospital environment. Furthermore, advanced assessment and management of patients with sepsis often requires a critical care environment. Thus effective care of patients with a diagnosis of sepsis requires effective co-ordination between the diagnosing 
Current research questions

1. Are there clinical, biochemical or microbiological markers that can be used to reliably differentiate patients with infection from patients with sepsis?

2. What individual aspects of the Surviving Sepsis Campaign bundle and Sepsis Six bundle have the greatest impact on outcomes?

3. What is the optimum volume of fluid resuscitation in patients with sepsis and septic shock?

\section{Main messages}

1. The quick Sequential (sepsis-related) Organ Failure Assessment Score (qSOFA) can be used by clinicians as a bedside tool to identify patients with infection who may have sepsis. qSOFA is positive if the patient has at least two of the following clinical criteria:

- Respiratory rate of $22 /$ min or greater,

- Altered mentation (Glasgow Coma Scale of <15)

- Systolic blood pressure of $100 \mathrm{~mm} \mathrm{Hg}$ or less.

2. Track and trigger early warning scores such as the MEWS and NEWS scores can also be used to identify deteriorating patients with infection who require rapid treatment for sepsis.

3. The clinical diagnosis of sepsis should trigger appropriate management bundles, such as the Sepsis Six bundle, to be completed within 1 hour of diagnosing sepsis:

- Administer oxygen to maintain $\mathrm{SpO}_{2}$ at $>94 \%$.

- Take blood cultures and consider infective source.

- Administer intravenous antibiotics.

- Consider intravenous fluid resuscitation.

- Check serial lactates.

- Commence hourly urine output measurement.

4. These simple steps will restore tissue hypoperfusion, treat the underlying infection and allow time for source identification, assessment of end organ damage and risk stratification (using the SOFA score), review of diagnosis and liaison with critical care teams. Hospitals should ensure that all medical staff are familiar with the diagnostic criteria for sepsis, the Surviving Sepsis Campaign guidelines and Sepsis Six bundles. Hospitals should also facilitate the implementation of these bundles through the use of screening tools and management pathways.

non-intensivist and the critical care team. However, the mainstay of effective management of sepsis is antibiotic therapy, intravenous fluid resuscitation to restore tissue perfusion, source control, and frequent re-evaluation by a clinician. These interventions can be delivered quickly and effectively in ordinary emergency departments or wards with the assistance of care pathways and protocols.

\section{SUMMARY}

While there is an ever-growing body of evidence informing the optimal management of sepsis in intensive care settings, non-intensivist medical staff will continue to play a key role in the early identification and management of these patients. It is therefore essential that all medical staff are well educated in the recognition and management of sepsis, and that systems are in place to ensure that this is done reliably.
Key references

- Rhodes A, Evans LE, Alhazzani W, et al. Surviving Sepsis Campaign: International Guidelines for Management of Sepsis and Septic Shock: 2016. Intensive Care Med 2017:43:1-74.

- Angus DC, Barnato AE, Bell D, et al. A systematic review and meta-analysis of early goal-directed therapy for septic shock: the ARISE, ProCESS and ProMISe Investigators. Intensive Care Med 2015;41:1549-60.

- Singer M, Deutschman CS, Seymour C, et al. The Third International Consensus Definitions for Sepsis and Septic Shock (Sepsis-3). JAMA 2016;315:801-10.

- Kumar A, Roberts D, Wood KE, et al. Duration of hypotension before initiation of effective antimicrobial therapy is the critical determinant of survival in human septic shock. Crit Care Med 2006;34:1589-96.

\section{Self assessment questions}

Please answer true or false to the below statements.

1. A 68-year-old man presents with a 3-day history of acute confusion, productive couch, dyspnoea and general malaise. His respiratory rate is 34 breaths per minute, his oxygen saturations are $96 \%$, his pulse is 104 beats per minute, his blood pressure is $90 / 40 \mathrm{~mm} \mathrm{Hg}$, and his temperature is $37.8^{\circ} \mathrm{C}$. His chest $\mathrm{x}$-ray shows left lobar consolidation. This is a case of pneumonia and sepsis.

2. A 49-year-old woman presents with abdominal pain, fever, rigours and is clinically jaundiced. Her respiratory rate is 30 breaths per minute, her oxygen saturations are $98 \%$, her pulse is 112 beats per minute, her blood pressure is $88 / 66 \mathrm{~mm} \mathrm{Hg}$ and her temperature is $39.0^{\circ} \mathrm{C}$. Her serum lactate measures $3.0 \mathrm{mmol} / \mathrm{L}$. She is resuscitated aggressively with intravenous fluids by the admitting junior doctor, who requests baseline laboratory investigations to confirm his suspicion of obstructive jaundice with cholangitis. Two hours later her results are available, by which time she has been in hospital for 3.5 hours without antibiotics. This delay is unlikely to have an impact on the patient's overall outcome.

3. Combined antibiotic therapy should always be first line in all cases where there is sepsis of unknown origin.

4. A 72-year-old female patient has symptoms of dysuria and fever. Her initial blood pressure was $66 / 42 \mathrm{~mm} \mathrm{Hg}$. She has received $2 \mathrm{~L}$ of intravenous fluid and broad spectrum antibiotics. Her blood pressure after 1 hour is $76 / 45 \mathrm{~mm} \mathrm{Hg}$. She should be referred by the non-intensivist to the intensive care team for initiation of vasopressor therapy.

5. A 48-year-old man is admitted with suspected meningitis. He has no significant past medical history. He has a blood pressure of $78 / 36 \mathrm{~mm} \mathrm{Hg}$. His serum lactate is $4.2 \mathrm{mmol} / \mathrm{L}$. He is referred to intensive care services. His serum glucose is $9.2 \mathrm{mmol} / \mathrm{L}$. He should be started on insulin infusion to keep his serum glucose below $6 \mathrm{mmol} / \mathrm{L}$.

Contributors EN planned and proposed the review. AK and PH drafted the initial paper. EN critically reviewed the draft, edited and submitted the final paper.

Competing interests None declared.

Provenance and peer review Not commissioned; externally peer reviewed.

(C) Article author(s) (or their employer(s) unless otherwise stated in the text of the article) 2017. All rights reserved. No commercial use is permitted unless otherwise expressly granted. 


\section{REFERENCES}

1 Angus DC, Linde-Zwirble WT, Lidicker J, et al. Epidemiology of severe sepsis in the United States: analysis of incidence, outcome, and associated costs of care. Crit Care Med 2001;29:1303-10.

2 Harrison DA, Welch CA, Eddleston JM. The epidemiology of severe sepsis in England, Wales and Northern Ireland, 1996 to 2004: secondary analysis of a high quality clinical database, the ICNARC Case Mix Programme Database. Crit Care 2006;10:R42.

3 Dombrovskiy VY, Martin AA, Sunderram J, et al. Rapid increase in hospitalization and mortality rates for severe sepsis in the United States: a trend analysis from 1993 to 2003. Crit Care Med 2007;35:1244-50.

4 Kumar G, Kumar N, Taneja A, et al. Nationwide trends of severe sepsis in the 21st century (2000-2007). Chest 2011;140:1223-31.

5 Dellinger RP, Levy MM, Rhodes A, et al. Surviving Sepsis Campaign: International Guidelines for Management of Severe Sepsis and Septic Shock: 2012. Crit Care Med 2013:41:580-637.

6 Vincent JL, Sakr Y, Sprung CL, et al. Sepsis in European intensive care units: results of the SOAP study. Crit Care Med 2006;34:344-53.

7 Lagu T, Rothberg MB, Shieh MS, et al. Hospitalizations, costs, and outcomes of severe Sepsis in the United States 2003 to 2007. Crit Care Med 2012:40:754-61.

8 Singer M, Deutschman CS, Seymour CW, et al. The Third International Consensus Definitions for Sepsis and Septic Shock (Sepsis-3). JAMA 2016;315:801-10.

9 Remick DG. Pathophysiology of sepsis. Am J Pathol 2007;170:1435-44.

10 Bosmann M, Ward PA. The inflammatory response in Sepsis. Trends Immunol 2013;34:129-36.

11 Dellinger RP, Levy MM, Carlet JM, et al. Surviving Sepsis Campaign: international guidelines for management of severe Sepsis and septic shock: 2008. Crit Care Med 2008;36:296-327.

12 Levy MM, Dellinger RP, Townsend SR, et al. The Surviving Sepsis Campaign: results of an international guideline-based performance improvement program targeting severe Sepsis. Intensive Care Med 2010;36:222-31.

13 Dellinger RP, Carlet JM, Masur H, et al. Surviving Sepsis Campaign guidelines for management of severe Sepsis and septic shock. Crit Care Med 2004:32:858-73.

14 Levy MM, Rhodes A, Phillips GS, et al. Surviving Sepsis Campaign: association between performance metrics and outcomes in a 7.5-year study. Crit Care Med 2015;43:3-12.

15 Rhodes A, Evans LE, Alhazzani W, et al. Surviving Sepsis Campaign: International Guidelines for Management of Sepsis and Septic Shock: 2016. Intensive Care Med 2017:43:304-77.

16 Rivers E, Nguyen B, Havstad S, et al. Early goal-directed therapy in the treatment of severe Sepsis and septic shock. N Engl J Med 2001;345:1368-77.

17 Yealy DM, Kellum JA, Huang DT, et al. A randomized trial of protocol-based care for early septic shock. N Engl J Med 2014;370:1683-93.

18 Peake SL, Delaney A, Bailey M, et al. Goal-directed resuscitation for patients with early septic shock. N Engl J Med 2014;371:1496-506

19 Mouncey PR, Osborn TM, Power GS, et al. Trial of early, goal-directed resuscitation for septic shock. N Engl J Med 2015;372:1301-11.

20 Barochia AV, Cui X, Vitberg D, et al. Bundled care for septic shock: an analysis of clinical trials. Crit Care Med 2010;38:668-78.

21 Surviving Sepsis Campaign. Surviving Sepsis Campaign Responds to Sepsis-3. http://w ww.survivingsepsis.org/SiteCollectionDocuments/SSC-Statements-Sepsis-Definitions3-2016.pdf (accessed Aug 2016).

22 Kaukonen KM, Bailey M, Pilcher D, et al. Systemic inflammatory response syndrome criteria in defining severe Sepsis. N Engl J Med 2015;372:1629-38.

23 Keep JW, Messmer AS, Sladden R, et al. National early warning score at emergency department triage may allow earlier identification of patients with severe Sepsis and septic shock: a retrospective observational study. Emerg Med J 2016;33:37-41.

24 Corfield AR, Lees F, Zealley l, et al. Utility of a single early warning score in patients with Sepsis in the emergency department. Emerg Med J 2014;31:482-7.

25 Churpek MM, Snyder A, Han X, et al. Quick Sepsis-related Organ Failure Assessment, Systemic Inflammatory Response Syndrome, and Early Warning Scores for Detecting Clinical Deterioration in Infected Patients outside the Intensive Care Unit. Am J Respir Crit Care Med 2017:195:906-911.

26 Daniels R. Surviving the first hours in sepsis: getting the basics right (an intensivist's perspective). J Antimicrob Chemother 2011;66(suppl 2):ii11-23.

27 Casserly B, Phillips GS, Schorr C, et al. Lactate measurements in sepsis-induced tissue hypoperfusion: results from the Surviving Sepsis Campaign database. Crit Care Med 2015:43:567-73.

28 Trzeciak S, Dellinger RP, Chansky ME, et al. Serum lactate as a predictor of mortality in patients with infection. Intensive Care Med 2007:33:970-7.

29 Hanudel P, Wilcox S, Cadin E, et al. 55: prevalence of cryptic shock in a cohort of outof-hospital sepsis patients: an argument for out-of-hospital point-of-care lactate. Ann Emerg Med 2008;51:487-8

30 Lee A, Mirrett S, Reller LB, et al. Detection of bloodstream infections in adults: how many blood cultures are needed? J Clin Microbiol 2007;45:3546-8.

31 Coburn B, Morris AM, Tomlinson G, et al. Does this adult patient with suspected bacteremia require blood cultures? JAMA 2012;308:502-11.
32 O'Brien DJ, Gould IM. Maximizing the impact of antimicrobial stewardship: the role of diagnostics, national and international efforts. Curr Opin Infect Dis 2013;26:352-8.

33 Gasser E, Schell-Chaple H. 1053: Impact of standardized algorithm on timeliness to blood culture collection in sepsis. Crit Care Med 2013:41:A265.

34 Mitteregger D, Barousch W, Nehr M, et al. Neutralization of antimicrobial substances in new BacT/Alert FA and FN Plus blood culture bottles. J Clin Microbiol 2013;51:1534-40

35 Michael B, Menezes BF, Cunniffe J, et al. Effect of delayed lumbar punctures on the diagnosis of acute bacterial meningitis in adults. Emerg Med J 2010;27:433-8.

36 Kumar A, Roberts D, Wood KE, et al. Duration of hypotension before initiation of effective antimicrobial therapy is the critical determinant of survival in human septic shock. Crit Care Med 2006;34:1589-96.

37 Ferrer R, Martin-Loeches I, Phillips G, et al. Empiric antibiotic treatment reduces mortality in severe Sepsis and septic shock from the first hour: results from a guideline-based performance improvement program. Crit Care Med 2014;42:1749-55

38 Rubinstein E, Keynan Y. Vancomycin-resistant enterococci. Crit Care Clin 2013;29:841-52

39 Lyle NH, Pena OM, Boyd JH, et al. Barriers to the effective treatment of sepsis: antimicrobial agents, sepsis definitions, and host-directed therapies. Ann N Y Acad Sci 2014;1323:101-14.

40 Perel P, Roberts I, Ker K. Colloids versus crystalloids for fluid resuscitation in critically ill patients. Cochrane Database Syst Rev 2013;2:CD000567.

41 Finfer S, Bellomo R, Boyce N, et al. A comparison of albumin and saline for fluid resuscitation in the intensive care unit. N Engl J Med 2004;350:2247-56.

42 Delaney AP, Dan A, McCaffrey J, et al. The role of albumin as a resuscitation fluid for patients with Sepsis: a systematic review and meta-analysis. Crit Care Med 2011:39:386-91.

43 Kelm DJ, Perrin JT, Cartin-Ceba R, et al. Fluid overload in patients with severe Sepsis and septic shock treated with early goal-directed therapy is associated with increased acute need for fluid-related medical interventions and hospital death. Shock 2015;43:68-73.

44 Schein M, Marshall J. Source control for surgical infections. World J Surg 2004;28:638-45

45 Marshall JC, Maier RV, Jimenez M, et al. Source control in the management of severe Sepsis and septic shock: an evidence-based review. Crit Care Med 2004;32(11 Suppl):S513-26.

46 Marshall JC, Al Naqbi A. Principles of source control in the management of Sepsis. Crit Care Nurs Clin North Am 2011;23:99-114.

47 Buck DL, Vester-Andersen M, Møller MH. Surgical delay is a critical determinant of survival in perforated peptic ulcer. Br J Surg 2013;100:1045-9.

48 Azuhata T, Kinoshita K, Kawano D, et al. Time from admission to initiation of surgery for source control is a critical determinant of survival in patients with gastrointestinal perforation with associated septic shock. Crit Care 2014;18:R87.

49 Angus DC, Barnato AE, Bell D, et al. A systematic review and meta-analysis of early goal-directed therapy for septic shock: the ARISE, ProCESS and ProMISe Investigators. Intensive Care Med 2015;41:1549-60.

50 Jansen TC, van Bommel J, Schoonderbeek FJ, et al. Early lactate-guided therapy in intensive care unit patients: a multicenter, open-label, randomized controlled trial. Am J Respir Crit Care Med 2010;182:752-61.

51 Arnold RC, Shapiro NI, Jones AE, et al. Multicenter study of early lactate clearance as a determinant of survival in patients with presumed Sepsis. Shock 2009;32:35-9.

52 Kruse 0, Grunnet N, Barfod C. Blood lactate as a predictor for in-hospital mortality in patients admitted acutely to hospital: a systematic review. Scand J Trauma Resusc Emerg Med 2011;19:74.

53 Jones AE, Shapiro NI, Trzeciak S, et al. Lactate clearance vs central venous oxygen saturation as goals of early Sepsis therapy: a randomized clinical trial. JAMA 2010;303:739-46.

54 Briegel J, Forst $\mathrm{H}$, Haller $\mathrm{M}$, et al. Stress doses of hydrocortisone reverse hyperdynamic septic shock: a prospective, randomized, double-blind, single-center study. Crit Care Med 1999:27:723-32.

55 Annane D, Sébille V, Charpentier C, et al. Effect of treatment with low doses of hydrocortisone and fludrocortisone on mortality in patients with septic shock. JAMA 2002;288:862-71.

56 Sprung CL, Annane D, Keh D, et al. Hydrocortisone therapy for patients with septic shock. N Engl J Med 2008;358:111-24.

57 Hébert PC, Wells G, Blajchman MA, et al. A multicenter, randomized, controlled clinical trial of transfusion requirements in critical care. $N$ Engl I Med Overseas Ed 1999:340:409-17.

58 Wilson M, Weinreb J, Hoo GW. Intensive insulin therapy in critical care: a review of 12 protocols. Diabetes Care 2007;30:1005-11.

59 Wiener RS, Wiener DC, Larson RJ. Benefits and risks of tight glucose control in critically ill adults: a meta-analysis. JAMA 2008;300:933-44.

60 Ling Y, Li X, Gao X. Intensive versus conventional glucose control in critically ill patients: a meta-analysis of randomized controlled trials. Eur J Intern Med 2012;23:564-74

61 Marik PE, Preiser JC. Toward understanding tight glycemic control in the ICU: a systematic review and metaanalysis. Chest 2010;137:544-51. 
62 Kansagara D, Fu R, Freeman M, et al. Intensive insulin therapy in hospitalized patients: a systematic review. Ann Intern Med 2011;154:268-82.

63 Finfer S, Chittock DR, Su SY, et al. Intensive versus conventional glucose control in critically ill patients. N Engl J Med 2009;360:1283-97.

64 Geerts W, Cook D, Selby R, et al. Venous thromboembolism and its prevention in critical care. J Crit Care 2002;17:95-104.

65 Marik PE, Vasu T, Hirani A, et al. Stress ulcer prophylaxis in the new millennium: a systematic review and meta-analysis. Crit Care Med 2010;38:2222-8.

66 Alhazzani W, Alenezi F, Jaeschke RZ, et al. Proton pump inhibitors versus histamine 2 receptor antagonists for stress ulcer prophylaxis in critically ill patients: a systematic review and meta-analysis. Crit Care Med 2013;41:693-705.

67 Cook DJ, Fuller HD, Guyatt GH, et al. Risk factors for gastrointestinal bleeding in critically ill patients. N Engl J Med Overseas Ed 1994;330:377-81.

68 Carlbom DJ, Rubenfeld GD. Barriers to implementing protocol-based sepsis resuscitation in the emergency department--results of a national survey. Crit Care Med 2007;35:2525-32.

69 Simmonds M, Hutchinson A, Chikhani M, et al. Surviving sepsis beyond intensive care: a retrospective cohort study of compliance with the International guidelines. $J$ Intensive Care Soc 2008;9:124-7.

\section{Answers}

1. True. There is evidence of infection (pneumonia) with a positive qSOFA score. The patient has a raised early warning score (eg, MEWS or NEWS score) of at least 5 as a result of tachypnoea, hypotension and tachycardia. The patient has signs of organ dysfunction and should be treated without delay for pneumonia and sepsis.

2. False. There is compelling evidence that each hour delay in the administration of intravenous antibiotics in sepsis with hypotension is associated with increased mortality.

3. False. Combined antibiotic therapy should be used as first line treatment in patients with known multi-drug resistant pathogens, in patients with septic shock and in patients with neutropaenia.

4. True.

5. False. There is evidence to suggest that outcomes are worse with tight glycaemic control, and therefore Surviving Sepsis Campaign recommends the use of an insulin protocol to maintain glucose at a cut-off value of $10 \mathrm{mmol} / \mathrm{L}$ in sepsis. 